\title{
Radiative and flavor-violating transitions of leptons from interactions with color-octet particles
}

\author{
Yi Liao ${ }^{a, b}, 1, \mathrm{Ji}^{-}$Yuan $\mathrm{Liu}^{b}$ \\ ${ }^{a}$ Center for High Energy Physics, Peking University, Beijing 100871, China \\ ${ }^{b}$ School of Physics, Nankai University, Tianjin 300071, China \\ ${ }^{c}$ Kavli Institute for Theoretical Physics China, CAS, Beijing 100190, China
}

\begin{abstract}
It has been recently proposed that neutrino mass could originate from Yukawa interactions of leptons with new colored particles. This raises the interesting possibility of testing mass generation through copious production of those particles at hadron colliders. A realistic assessment of it however should take into account how large those interactions could be from available precision results. In this work we make a systematic analysis to the flavor structure in Yukawa couplings, provide a convenient parametrization to it, and investigate the rare radiative and pure leptonic decays of the muon and tau leptons. For general values of parameters the muon decays set stringent constraints on the couplings, and all rare tau decays are far below the current experimental sensitivity. However, there is room in parameter space in which the muon decays could be significantly suppressed by destructive interference between colored particles without generically reducing the couplings themselves. This is also the region of parameters that is relevant to collider physics. We show that for this part of parameter space some tau decays can reach or are close to the current level of precision.
\end{abstract}

PACS: 14.60.Pq, 13.35.-r, 13.15.+g

Keywords: radiative neutrino mass, color octet particles, rare lepton decays

${ }^{1}$ liaoy@nankai.edu.cn 


\section{Introduction}

It has been verified by oscillation and other low energy experiments that neutrinos have tiny and non-degenerate masses, yet the mechanism for mass generation has remained mysterious. It is generally believed that the new physics relevant to the tiny mass lies beyond the standard model (SM) and its effects may be systematically accounted for by high dimensional operators. Indeed, viewing SM as an effective field theory at low energies one can write down a unique dimension five operator in terms of the Higgs and lepton doublet fields that generates the neutrino mass upon spontaneous breaking of electroweak symmetries [1]. It is interesting that there are only three realizations of the operator at tree level [2] which correspond exactly to the three types of seesaw models [3, 4, 5]. In each case, a single representation under the SM gauge group, $S U(3)_{C} \times S U(2)_{L} \times U(1)_{Y}$, is prescribed for the new fields to link the Higgs and lepton doublets.

In the above seesaw models, the tininess of the neutrino mass is attributed to a huge scale of new physics or feeble interactions that induce the operator. In either case it would be difficult to detect low energy effects of new physics beyond neutrino mass. One way to relieve the tension is to consider certain radiative origin of neutrino mass [6]. By prescribing two or more types of representations but excluding those utilized in the three seesaw models, it is possible to generate tiny neutrino mass without requiring all couplings to be diminishingly small or all new particles to be inaccessibly heavy. This is even so when the mass originates from a two-loop [7, 8] or three-loop effect [9]. These radiative mechanisms usually employ small representations of the electroweak group. The other way to generate neutrino mass without sacrificing too much in couplings or heavy masses has been suggested recently [10]. By assigning larger representations, it is possible to forbid the dimension five operator at tree level so that the operator relevant for neutrino mass first appears at dimension seven.

In all of the above mechanisms it has been tacitly assumed that the particles responsible for neutrino mass generation do not participate strong interactions. But this could well be an easy prejudice as there is no experimental hint for it at all. The idea that neutrino mass may originate from interactions with colored particles becomes especially relevant now. These particles if not very heavy can be copiously produced at the LHC so that the origin of neutrino mass could potentially be tested there. A concrete model in this spirit has been recently proposed by Fileviez Perez and Wise [11]. Since the colored fields must appear in pair in interactions responsible for neutrino mass, the minimal choice includes both scalar and fermionic degrees of freedom. To avoid chiral anomaly from the new fermions, they considered the simplest option that the fermions belong to the adjoint representation of $S U(3)_{C}$ and are neutral under $U(1)_{Y}$. This in turn singles out naturally the octet scalars that were previously introduced in the quark sector [12, 13] in the framework of minimal flavor violation [14]. The potential relevance of the model to leptogenesis has been discussed in Ref. [15].

The interactions that generate neutrino mass and mixing generically mediate lepton flavor violating (LFV) transitions and anomalous magnetic moments of charged leptons. While the measurements in the $e \mu$ sector have reached an impressive level of precision [16, 17, 18, 19], the upper bounds on LFV decays of the tau lepton are improving rapidly at the $B$ factories, see Refs. 
[20, 21, 22, 23] for the precision frontier. And even more stringent limits on some of them are expected in the near future. These constraints from low energy processes will significantly affect the feasibility of testing neutrino mass mechanisms at colliders. The purpose of the current work is to investigate systematically those processes in the color octet model suggested in Ref. [11], and to see if there is any room in the parameter space that could be relevant to collider physics. The LFV decays have been extensively studied in various models of neutrino mass and mixing. As a few examples, we mention Refs. [24] in supersymmetric models, [25, 26, 27] in seesaw models, [28] in a model of mirror fermions [29], and in [30] in a little Higgs model. We refer to the reviews [31] for a more complete list of references.

The paper is organized as follows. In the next section we introduce the octet model and provide a convenient parametrization to the Yukawa couplings. The radiative and pure leptonic transitions are calculated analytically in section 3, and the parameter space is then discussed in section 4. We summarize our main results in the last section. Some phase space integrals are evaluated in Appendix A.

\section{Parametrization of couplings in octet model}

As we briefly reviewed in section 1, the octet model [11] introduces the color-octet scalars and fermions on top of the SM Higgs field $H$ and lepton fields, $L_{L \alpha}, \ell_{R \alpha}$, where $L(R)$, $\alpha$ refer to chirality and flavor respectively. The octet scalars, $S_{a r}$, have the same quantum numbers under $S U(2)_{L} \times U(1)_{Y}$ as $H$. Here $a$ is the color index and $r$ enumerates the scalars. The octet fermions are neutral under $U(1)_{Y}$ but may be a singlet or triplet of $S U(2)_{Y}$, which we denote as the two cases:

$$
\begin{array}{ll}
\text { case A: } & \rho_{a x}, \\
\text { case B: } & \chi_{a x}=\left(\begin{array}{cc}
\frac{1}{\sqrt{2}} \chi_{a x}^{0} & \chi_{a x}^{+} \\
\chi_{a x}^{-} & -\frac{1}{\sqrt{2}} \chi_{a x}^{0}
\end{array}\right),
\end{array}
$$

where $x$ enumerates the fermions. We assume arbitrarily $\rho$ and $\chi$ are right-handed. While $\rho$ and $\chi^{0}$ are Majorana fields, $\chi^{ \pm}$being real under $S U(3)_{C}$ will be paired into a Dirac field. The Yukawa couplings of leptons in SM and the additional terms in the octet model are

$$
\begin{aligned}
-\mathscr{L}_{\mathrm{SM}}^{\mathrm{Yuk}} & =y_{\alpha \beta} \overline{L_{L \alpha}} H \ell_{R \beta}+\text { h.c. } \\
-\mathscr{L}_{\mathrm{A}}^{\mathrm{Yuk}} & =z_{\alpha x}^{r} \overline{L_{L \alpha}} \tilde{S}_{a r} \rho_{a x}+\text { h.c. }, \\
-\mathscr{L}_{\mathrm{B}}^{\text {Yuk }} & =z_{\alpha x}^{r} \overline{L_{L \alpha}} \chi_{a x} \tilde{S}_{a r}+\text { h.c. },
\end{aligned}
$$

where $\tilde{S}_{a r}=i \tau_{2} S_{a r}^{*}$ and summation over repeated indices is implied.

The charged leptons become massive when $H$ develops a vacuum expectation value, $\mathrm{v} / \sqrt{2}$. Since neutrinos are massless at tree level, the diagonalizing matrices for $\ell$ can be absorbed by redefinition of the fields. We assume this has been done already. For generally non-degenerate scalars or fermions there is no symmetry mixing them. Since the octet fermions are neutral 


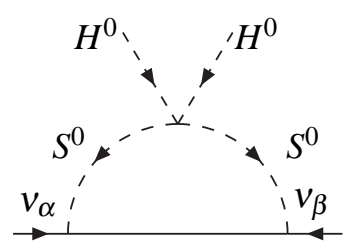

(a)

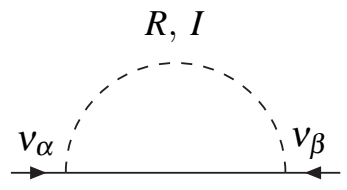

(b)

Figure 1: Diagrams for neutrino masses.

under $U(1)_{Y}$ and either an adjoint or a singlet of $S U(2)_{L}$, rephasing is not possible for them. For the scalars, we assume their phase convention has been fixed in the potential [12]. Thus what we can do at most is to rephase the fields $\ell_{L}$ (and $\ell_{R}$ identically), leaving the remaining magnitudes and phases in $z$ being physical parameters.

The neutrinos may gain mass from radiative corrections. Since there are no further degrees of freedom for them to pair with, the mass must necessarily be of Majorana nature. For this to be possible, the lepton number conservation has to be violated. This happens when the Yukawa couplings are augmented by the following term in the potential with a single octet scalar [12]:

$$
-V \supset-\frac{1}{2} \lambda\left(S_{a}^{\dagger} H\right)^{2}+\text { h.c. }
$$

where $\lambda$ is real by convention. It turns out that for two out of the three neutrinos to become massive, the minimal choice is to have either one octet scalar plus two octet fermions or the other way around [11]. To avoid the complicated mixing amongst scalars, we shall stick here to the former choice. We shall drop from now on the index $r$ while $x$ assumes values 1,2 . In this minimal scenario, the $z$ couplings contain nine physical parameters, i.e., six magnitudes plus three phases, and will be parameterized later in this section.

The neutrino mass matrix may be obtained by first computing the dimension five operator, $\overline{v_{\beta}} P_{L} v_{\alpha} H^{0} H^{0}$, depicted in Fig. 1(a), where the virtual fermion can be $\rho_{x}$ (case A) or $\chi_{x}^{0}$ (case B). The result is [11],

$$
M_{\alpha \beta}^{v}=\sum_{x=1}^{2} z_{\alpha x}^{*} z_{\beta x}^{*} \mu_{x}
$$

where

$$
\mu_{x}=\xi C \frac{\lambda \mathrm{v}^{2} m_{x}}{32 \pi^{2} m_{0}^{2}} \frac{1+r_{x}\left(\ln r_{x}-1\right)}{\left(r_{x}-1\right)^{2}}, r_{x}=\frac{m_{x}^{2}}{m_{0}^{2}}
$$

Here $C=N_{C}^{2}-1=8$ is a color factor, and $\xi=1(1 / 2)$ in case A ( B). We have denoted the masses of $S^{0}$ and $\rho_{x}\left(\chi_{x}^{0}\right)$ by $m_{0}$ and $m_{x}$ respectively. Since the mass splitting between the neutral and charged octet particles arises at a higher order, we shall ignore it and use the same notation for $S^{ \pm}$(and $\chi_{x}^{ \pm}$in case B). The mass matrix can also be obtained by calculating 
the self-energy of neutrinos at zero momentum shown in Fig. 1(b), where the decomposition $S_{a}^{0}=\left(R_{a}+i I_{a}\right) / \sqrt{2}$ has been made. The result is to replace the above $\mu_{x}$ by the following one:

$$
\mu_{x}=\frac{\xi C}{32 \pi^{2}} \frac{m_{x}}{\left(m_{x}^{2}-m_{R}^{2}\right)\left(m_{x}^{2}-m_{I}^{2}\right)}\left[m_{R}^{2} m_{x}^{2} \ln \frac{m_{x}^{2}}{m_{R}^{2}}-m_{I}^{2} m_{x}^{2} \ln \frac{m_{x}^{2}}{m_{I}^{2}}+m_{R}^{2} m_{I}^{2} \ln \frac{m_{R}^{2}}{m_{I}^{2}}\right],
$$

where $m_{R, I}$ are the masses of $R, I$, whose splitting is measured by the $\lambda$ term in the potential,

$$
m_{R}^{2}-m_{I}^{2}=\lambda \mathrm{v}^{2}
$$

The result in eq (5) is an approximation to eq (6) in the limit of small mass splitting, $\left|\lambda \mathrm{v}^{2}\right| \ll$ $\left(m_{R}^{2}+m_{I}^{2}\right) / 2$. Since $\lambda$ is a measure of lepton number violation, it should be naturally small. We shall work in this limit below.

With two octet fermions the matrix $M^{v}$ is degenerate and has a zero eigenvalue. This arises because it is a product of a matrix of lower rank with its transpose. Noting $\mu_{x} / \lambda>0$ for arbitrary masses, we can express it as $M^{v}=Z Z^{T}$, where $Z=\left(\mathbf{u}_{1}^{*}, \mathbf{u}_{2}^{*}\right)$ is a $3 \times 2$ matrix in terms of the column vectors, $\mathbf{u}_{x}=\sqrt{\mu_{x}} z_{\alpha x}$ (not summed over $x$ ) for $\lambda>0$, or $\mathbf{u}_{x}=\sqrt{-\mu_{x}}\left(i z_{\alpha x}\right)$ for $\lambda<0$. The factors of $i$ in the latter case will not affect our later results which involve $z$ always in the form of $z_{\alpha x}^{*} z_{\beta x}$, and can thus be ignored. This implies that we can restrict ourselves in phenomenological analysis to $\lambda>0$ without loss of generality. Diagonalization by the leptonic mixing matrix $U_{\text {PMNS }}$ yields two possible spectra of normal or inverted hierarchy:

$$
\begin{aligned}
\mathrm{NH}: & m_{v_{1}}=0, m_{v_{2}}=\lambda_{-}, m_{v_{3}}=\lambda_{+}, \\
\mathrm{IH}: & m_{v_{3}}=0, m_{v_{1}}=\lambda_{-}, m_{v_{2}}=\lambda_{+},
\end{aligned}
$$

where $\lambda_{+}>\lambda_{-}>0$ are the two non-zero eigenvalues. For both hierarchies, we have $U_{\text {PMNS }}=$ $U_{\mathrm{D}} E_{v}$, where $U_{\mathrm{D}}$ is the analog of the CKM matrix with three angles $\left(\theta_{12}, \theta_{23}, \theta_{13}\right)$ and a Dirac $\mathrm{CP}$ phase $\delta$, and $E_{V}=\operatorname{diag}\left(1, e^{i \alpha}, 1\right)$ contains a single Majorana phase. We write in terms of the column vectors:

$$
U_{\mathrm{PMNS}}=\left(\mathbf{x}_{1}, \mathbf{x}_{2}, \mathbf{x}_{3}\right),
$$

with $\mathbf{x}_{i}^{\dagger} \mathbf{x}_{j}=\delta_{i j}$. It can be readily verified that the zero eigenvalue requires the corresponding row (first for $\mathrm{NH}$ or third for $\mathrm{IH}$ ) of $U_{\mathrm{PMNS}}^{T} Z$ to vanish. This means that $Z$ can be generally parameterized in terms of the other two column vectors in $U_{\mathrm{PMNS}}$ :

$$
\begin{aligned}
\mathrm{NH}: & \mathbf{u}_{1}=c_{-} \mathbf{x}_{2}+c_{+} \mathbf{x}_{3}, \mathbf{u}_{2}=d_{-} \mathbf{x}_{2}+d_{+} \mathbf{x}_{3}, \\
\mathrm{IH}: & \mathbf{u}_{1}=c_{-} \mathbf{x}_{1}+c_{+} \mathbf{x}_{2}, \mathbf{u}_{2}=d_{-} \mathbf{x}_{1}+d_{+} \mathbf{x}_{2} .
\end{aligned}
$$

The coefficients $c_{ \pm}, d_{ \pm}$can be determined in terms of the eigenvalues $\lambda_{ \pm}$and a free complex parameter. We find that the following parametrization is convenient and applies to both hierarchies:

$$
\begin{aligned}
& c_{-}=\sqrt{\lambda_{-}} \frac{2 t}{1+t^{2}}, d_{-}=\sqrt{\lambda_{-}} \frac{1-t^{2}}{1+t^{2}} \\
& c_{+}=\sqrt{\lambda_{+}} \frac{1-t^{2}}{1+t^{2}}, d_{+}=\sqrt{\lambda_{+}} \frac{-2 t}{1+t^{2}}
\end{aligned}
$$




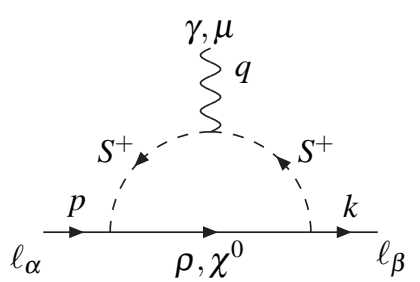

(a)

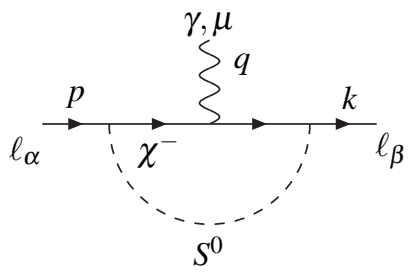

(d)

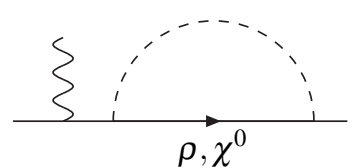

(b)

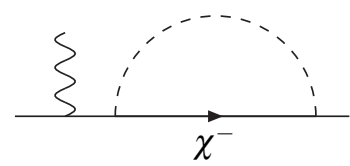

(e)

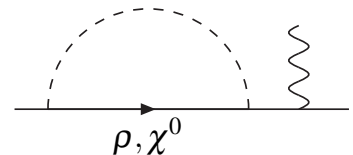

(c)

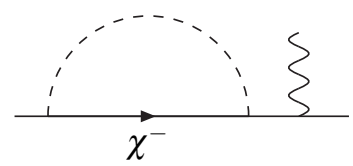

(f)

Figure 2: Diagrams for radiative transitions.

where $t$ is complex. And the Yukawa couplings now become

$$
z_{\alpha x}=\frac{\mathbf{u}_{\alpha x}}{\sqrt{\left|\mu_{x}\right|}}
$$

The physical parameters in $z_{\alpha x}$ have been traded for two neutrino masses $\lambda_{ \pm}$, three angles $\theta_{i j}$, one Dirac phase $\delta$, one Majorana phase $\alpha$, and one complex number $t$.

The diagonalization of leptons causes no other changes in gauge interactions but attaching $U_{\text {PMNS }}$ to their charged currents. In particular, the neutral currents of leptons are flavor diagonal. We can therefore restrict ourselves to interactions involving octet particles for the effects that are suppressed by SM interactions. Introducing the four-component fields,

$$
v=\left(\begin{array}{c}
v_{L} \\
v_{L}^{C}
\end{array}\right), \rho_{a}=\left(\begin{array}{c}
\rho_{a R}^{C} \\
\rho_{a R}
\end{array}\right), \chi_{a}^{0}=\left(\begin{array}{c}
\chi_{a R}^{0 C} \\
\chi_{a R}^{0}
\end{array}\right), \chi_{a}^{-}=\left(\begin{array}{c}
\chi_{a R}^{+C} \\
\chi_{a R}^{-}
\end{array}\right),
$$

and using $\bar{f} g=\overline{g^{C}} f^{C}$, the Yukawa couplings are modified to $\left(P_{R}=\left(1+\gamma_{5}\right) / 2\right)$,

$$
\begin{aligned}
-\mathscr{L}_{\mathrm{A}}^{\mathrm{Yuk}}= & \bar{v} U_{\mathrm{PMNS}}^{\dagger} z P_{R} \rho_{a} S_{a}^{0 *}-\bar{\ell} z P_{R} \rho_{a} S_{a}^{-}+\text {h.c. } \\
-\mathscr{L}_{\mathrm{B}}^{\mathrm{Yuk}}= & \frac{1}{\sqrt{2}} \bar{v} U_{\mathrm{PMNS}}^{\dagger} z P_{R} \chi_{a}^{0} S_{a}^{0 *}-\overline{\chi_{a}^{-}}\left(U_{\mathrm{PMNS}}^{\dagger} z\right)^{T} P_{R} v S_{a}^{-} \\
& +\bar{\ell} z P_{R} \chi_{a}^{-} S_{a}^{0 *}+\frac{1}{\sqrt{2}} \bar{\ell} z P_{R} \chi_{a}^{0} S_{a}^{-}+\text {h.c.. }
\end{aligned}
$$

Note in passing that the massless neutrino does not couple to color octet fermions.

\section{Radiative and pure leptonic transitions}

A direct consequence of the interactions shown in eq (14) is the occurrence of LFV transitions of the charged leptons, $\ell_{\alpha} \rightarrow \ell_{\beta} \gamma$ and $\ell_{\delta} \rightarrow \ell_{\alpha} \ell_{\beta} \bar{\ell}_{\gamma}$, and the contribution to related quantities 
like the anomalous magnetic moment and the muonium-anti-muonium transition rate. We start with the transition $\ell_{\alpha}(p) \rightarrow \ell_{\beta}(k)+\gamma^{(*)}(q)$, which also contributes to the pure leptonic decay when the photon converts to a pair of leptons. Our sign convention for the QED coupling is, $\mathscr{L}_{\mathrm{QED}}=-e A_{\mu} \bar{\ell} \gamma^{\mu} \ell$.

The Feynman diagrams are shown in Fig. 2, where (a)-(c) appear in both cases A and B and (d)-(f) occur only for case B. Working to the first nontrivial order in the external momenta, a straightforward calculation yields the amplitude:

$$
\begin{aligned}
-\frac{(4 \pi)^{2} m_{0}^{2}}{C e} \mathscr{A}_{\mu}= & z_{\beta x} z_{\alpha x}^{*}\left\{\left[\xi F_{1}\left(r_{x}\right)+2(1-\xi) G_{1}\left(r_{x}\right)\right] \bar{u}(k) P_{R}\left(q^{2} \gamma_{\mu}-q_{\mu} \phi\right) u(p)\right. \\
& \left.+\left[\xi F_{2}\left(r_{x}\right)+2(1-\xi) G_{2}\left(r_{x}\right)\right] \bar{u}(k)\left(m_{\alpha} P_{R}+m_{\beta} P_{L}\right) i \sigma_{\mu v} q^{v} u(p)\right\},
\end{aligned}
$$

where summation over $x$ is implied, and the loop functions are

$$
\begin{aligned}
F_{1}(x) & =\frac{1}{36(x-1)^{4}}\left[2-9 x+18 x^{2}-11 x^{3}+6 x^{3} \ln x\right], \\
F_{2}(x) & =\frac{1}{12(x-1)^{4}}\left[1-6 x+3 x^{2}+2 x^{3}-6 x^{2} \ln x\right], \\
G_{1}(x) & =\frac{1}{36(x-1)^{4}}\left[-16+45 x-36 x^{2}+7 x^{3}-12 \ln x+18 x \ln x\right], \\
G_{2}(x) & =\frac{1}{12(x-1)^{4}}\left[-2-3 x+6 x^{2}-x^{3}-6 x \ln x\right] .
\end{aligned}
$$

Some comments are in order. That the above Lorentz structures satisfy Ward identity serves as a useful check to our calculation. The $F_{1,2}$ terms from Figs (2a)-(2c) occur for both cases A and B while the $G_{1,2}$ terms from Figs (2d)-(2f) appear only in case B. This is controlled by the switch parameter $\xi$ introduced earlier. Since we have ignored the mass splitting between the neutral and charged particles, the loop functions depend only on the mass ratios of the fermions to the scalar. While $F_{1,2}(x), G_{1}(x)>0$ and $G_{2}(x)<0$, they all decrease monotonically in magnitude as $x$ increases. Since the contributions from the real and imaginary parts of $S^{0}$ in Figs. (2d)-(2f) simply add, when their mass splitting is relevant the exact result can be obtained by averaging the shown one over their masses.

From the above result we obtain the lepton anomalous magnetic moment and the branching ratio for the transition $\ell_{\alpha} \rightarrow \ell_{\beta} \gamma$ :

$$
\begin{aligned}
a\left(\ell_{\alpha}\right)= & \frac{1}{\pi} \frac{m_{\alpha}^{2}}{m_{0}^{2}} \sum_{x=1}^{2}\left|z_{\alpha x}\right|^{2}\left[\xi F_{2}\left(r_{x}\right)+2(1-\xi) G_{2}\left(r_{x}\right)\right] \\
\operatorname{Br}\left(\ell_{\alpha} \rightarrow \ell_{\beta} \gamma\right)= & \operatorname{Br}\left(\ell_{\alpha} \rightarrow v_{\alpha} \ell_{\beta} \bar{v}_{\beta}\right) \\
& \times \frac{12 \alpha_{\mathrm{EM}}}{\pi G_{F}^{2} m_{0}^{4}}\left|\sum_{x=1}^{2} z_{\beta x} z_{\alpha x}^{*}\left[\xi F_{2}\left(r_{x}\right)+2(1-\xi) G_{2}\left(r_{x}\right)\right]\right|^{2},
\end{aligned}
$$

where $\alpha_{\mathrm{EM}}$ is the fine structure constant. We have used $\Gamma\left(\ell_{\alpha} \rightarrow v_{\alpha} \ell_{\beta} \bar{v}_{\beta}\right)=G_{F}^{2} m_{\alpha}^{5} /\left(192 \pi^{3}\right)$ and neglected the final state masses in phase space integration. The decay $\mu \rightarrow e \gamma$ was also computed for case A (i.e., $\xi=1$ ) in Ref. [15], but our result differs from theirs. 


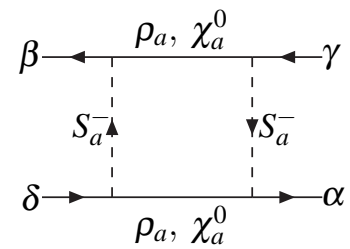

(a)

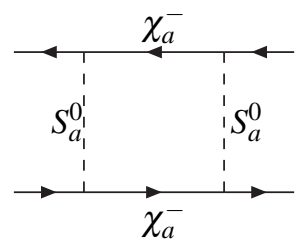

(b)

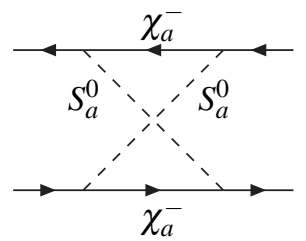

(c)

Figure 3: Additional box diagrams for pure leptonic decays.

The pure leptonic decay $\ell_{\delta}(p) \rightarrow \ell_{\alpha}\left(k_{1}\right) \ell_{\beta}\left(k_{2}\right) \bar{\ell}_{\gamma}\left(k_{3}\right)$ receives a contribution from box diagrams, in addition to the one from the off-shell radiative transition computed above when $\ell_{\beta}=\ell_{\gamma}$ or $\ell_{\alpha}=\ell_{\gamma}$. The box diagrams are shown in Fig. 3, where the arrows indicate the flow of negative charge. Fig. 3(a) appears for both cases A and B while Fig. 3(b) and (c) occur only for case B. In the approximation of neglecting the external momenta compared with the heavy octet masses, Fig. 3(b) and (c) cancel each other, leaving us with the result:

$$
\text { Fig. } 3=\frac{C \xi^{2}}{m_{0}^{2} i(4 \pi)^{2}} z_{\alpha x} z_{\delta x}^{*} z_{\beta y} z_{\gamma y}^{*} \bar{u}\left(k_{1}\right) \gamma_{\mu} P_{L} u(p) \bar{u}\left(k_{2}\right) \gamma^{\mu} P_{L} v\left(k_{3}\right) H\left(r_{x}, r_{y}\right) \text {, }
$$

where summations over $x$ and $y$ are implied, and the loop function is

$$
H(x, y)=\frac{1}{4(x-y)}\left[\frac{1}{1-x}-\frac{x \ln x}{1-x}+\frac{x \ln x}{(1-x)^{2}}-\frac{1}{1-y}+\frac{y \ln y}{1-y}-\frac{y \ln y}{(1-y)^{2}}\right],
$$

which is symmetric and positive-definite. The result has the correct anti-symmetry under the interchanges $\alpha \leftrightarrow \beta$ and $k_{1} \leftrightarrow k_{2}$ upon using Fierz identity.

With three generations of leptons there are three possible flavor configurations for the final states from a fixed initial state:

$$
\begin{aligned}
& \ell_{\delta}(p) \rightarrow \ell_{\alpha}\left(k_{1}\right) \ell_{\alpha}\left(k_{2}\right) \bar{\ell}_{\gamma}\left(k_{3}\right), \ell_{\alpha} \neq \ell_{\gamma}, \\
& \ell_{\delta}(p) \rightarrow \ell_{\alpha}\left(k_{1}\right) \ell_{\beta}\left(k_{2}\right) \bar{\ell}_{\beta}\left(k_{3}\right), \ell_{\alpha} \neq \ell_{\beta}, \\
& \ell_{\delta}(p) \rightarrow \ell_{\alpha}\left(k_{1}\right) \ell_{\alpha}\left(k_{2}\right) \bar{\ell}_{\alpha}\left(k_{3}\right) .
\end{aligned}
$$

We list their decay amplitudes separately. For decay (1), only the box diagram contributes:

$$
i \mathscr{A}(1)=\frac{2 C \xi^{2}}{m_{0}^{2} i(4 \pi)^{2}} z_{\alpha x} z_{\delta x}^{*} z_{\alpha y} z_{\gamma y}^{*} \bar{u}_{1} \gamma_{\mu} P_{L} u(p) \bar{u}_{2} \gamma^{\mu} P_{L} v_{3} H\left(r_{x}, r_{y}\right),
$$

where the factor 2 takes into account the identical particles in the final state upon using Fierz identity. For decay (2), both radiative and box diagrams contribute. It is important to make sure that their relative sign is correct:

$$
i \mathscr{A}(2)=i \mathscr{A}_{\triangle}+i \mathscr{A} \square
$$


where upon using equations of motion and Fierz identity and denoting $s_{i j}=\left(k_{i}+k_{j}\right)^{2}$,

$$
\begin{aligned}
i \mathscr{A}_{\triangle}= & \frac{-C e^{2} z_{\alpha x} z_{\delta x}^{*}}{m_{0}^{2} i(4 \pi)^{2}} \bar{u}_{2} \gamma^{\mu} v_{3} \bar{u}_{1}\left[P_{R} \gamma_{\mu}\left(\xi F_{1}\left(r_{x}\right)+2(1-\xi) G_{1}\left(r_{x}\right)\right)\right. \\
& \left.+\left(m_{\delta} P_{R}+m_{\alpha} P_{L}\right) i \sigma_{\mu v}\left(k_{2}+k_{3}\right)^{v} s_{23}^{-1}\left(\xi F_{2}\left(r_{x}\right)+2(1-\xi) G_{2}\left(r_{x}\right)\right)\right] u(p), \\
i \mathscr{A} \square= & \frac{C \xi^{2}}{m_{0}^{2} i(4 \pi)^{2}} z_{\beta y}^{*} z_{\delta x}^{*}\left(z_{\alpha x} z_{\beta y}+z_{\beta x} z_{\alpha y}\right) \bar{u}_{1} \gamma_{\mu} P_{L} u(p) \bar{u}_{2} \gamma^{\mu} P_{L} v_{3} H\left(r_{x}, r_{y}\right) .
\end{aligned}
$$

Finally, the amplitude for decay (3) can be obtained from eq (24) by substitutions and antisymmetrization:

$$
\mathscr{A}(3)=\left.\mathscr{A}(2)\right|_{\beta=\alpha}-\left(k_{1} \leftrightarrow k_{2}\right) .
$$

The branching ratio for the process (1) can be worked out straightforwardly using the phase space integral $I_{3}$ defined and calculated in Appendix A,

$$
\operatorname{Br}(1)=\operatorname{Br}\left(\ell_{\delta} \rightarrow \ell_{\alpha} v_{\delta} \bar{v}_{\alpha}\right) \frac{C^{2} \xi^{4}}{2^{10} \pi^{4} m_{0}^{4} G_{F}^{2}}\left|\sum_{x, y} z_{\alpha x} z_{\delta x}^{*} z_{\alpha y} z_{\gamma y}^{*} H\left(r_{x}, r_{y}\right)\right|^{2} .
$$

The decay rate for the process (2) is more complicated, both because it has radiative and box contributions and because there is a logarithmic singularity in the mass $\left(m_{\beta}\right)$ of leptons connected to the virtual photon. Care must be exercised in dropping $m_{\beta}$ in order not to miss terms at the considered order. We find it convenient to decompose the amplitude in the form:

$$
\begin{aligned}
C^{-1} m_{0}^{2}(4 \pi)^{2} \mathscr{A}(2)= & \left(B+T_{1}-T_{2} m_{\delta}^{2} s_{23}^{-1}\right) \bar{u}_{2} \gamma^{\mu} P_{L} v_{3} \bar{u}_{1} \gamma_{\mu} P_{L} u(p) \\
& +\left(T_{1}-T_{2} m_{\delta}^{2} s_{23}^{-1}\right) \bar{u}_{2} \gamma^{\mu} P_{R} v_{3} \bar{u}_{1} \gamma_{\mu} P_{L} u(p) \\
& +2 T_{2} m_{\delta} s_{23}^{-1} \bar{u}_{2} k_{1} v_{3} \bar{u}_{1} P_{R} u(p),
\end{aligned}
$$

where

$$
\begin{aligned}
B & =-\xi^{2} z_{\beta y}^{*} z_{\delta x}^{*}\left(z_{\alpha x} z_{\beta y}+z_{\beta x} z_{\alpha y}\right) H\left(r_{x}, r_{y}\right), \\
T_{1} & =e^{2} z_{\alpha x} z_{\delta x}^{*}\left(\xi F_{1}\left(r_{x}\right)+2(1-\xi) G_{1}\left(r_{x}\right)\right), \\
T_{2} & =e^{2} z_{\alpha x} z_{\delta x}^{*}\left(\xi F_{2}\left(r_{x}\right)+2(1-\xi) G_{2}\left(r_{x}\right)\right) .
\end{aligned}
$$

The decay rate is, in terms of the integrals in Appendix A,

$$
\begin{aligned}
\frac{\Gamma(2)}{m_{\delta}} \frac{m_{0}^{4}(4 \pi)^{4}}{C^{2} m_{\delta}^{4}}= & \left|B+T_{1}\right|^{2} 4 I_{3}+\left|T_{1}\right|^{2} 4 I_{3}-\operatorname{Re}\left(B T_{2}^{*}\right) 4 I_{2}-\operatorname{Re}\left(T_{1} T_{2}^{*}\right) 4 I_{1} \\
& +\left|T_{2}\right|^{2}\left[2 J_{1}-8 J_{2}+4 K\right],
\end{aligned}
$$

and the branching ratio is

$$
\begin{aligned}
\operatorname{Br}(2)= & \operatorname{Br}\left(\ell_{\delta} \rightarrow \ell_{\alpha} v_{\delta} \bar{v}_{\alpha}\right) \frac{C^{2}}{2^{11} \pi^{4} m_{0}^{4} G_{F}^{2}}\left\{\left|B+T_{1}\right|^{2}+\left|T_{1}\right|^{2}-4 \operatorname{Re}\left(B T_{2}^{*}\right)\right. \\
& \left.-8 \operatorname{Re}\left(T_{1} T_{2}^{*}\right)+\left[-\frac{14}{3}+8 \ln \frac{m_{\delta}^{2}}{4 m_{\beta}^{2}}\right]\left|T_{2}\right|^{2}\right\}
\end{aligned}
$$


Although the amplitude for the process (3) contains twice as many terms as for the process (2), its decay rate is not much more difficult to calculate. Each of the two terms in eq (25) when squared separately contributes the same to the rate, i.e., same as shown in eq (29) upon setting $\beta=\alpha$ and including a factor $\frac{1}{2}$ for identical particles. Although their interference has both $s_{23}^{-1}$ and $s_{13}^{-1}$ terms, the potential singularities do not overlap in kinematics. We can therefore do algebra with $m_{\alpha}=0$ without losing singular terms of the form $\ln m_{\alpha}^{2}$. Using the symmetry of phase space we find all those terms are cancelled in the interference, yielding a regular contribution to the rate. The sum is

$$
\begin{aligned}
\frac{\Gamma(3)}{m_{\delta}} \frac{m_{0}^{4}(4 \pi)^{4}}{C^{2} m_{\delta}^{4}}= & \left|B+T_{1}\right|^{2} 8 I_{3}+\left|T_{1}\right|^{2} 4 I_{3}-\operatorname{Re}\left(B T_{2}^{*}\right) 8 I_{2}-\operatorname{Re}\left(T_{1} T_{2}^{*}\right) 4\left(I_{1}+I_{2}\right) \\
& +\left|T_{2}\right|^{2}\left[2 I_{2}+2 J_{1}-8 J_{2}+4 K\right]
\end{aligned}
$$

where $B, T_{1}$, and $T_{2}$ are obtained from eq (28) by setting $\beta=\alpha$. The branching ratio is,

$$
\begin{aligned}
\operatorname{Br}(3)= & \operatorname{Br}\left(\ell_{\delta} \rightarrow \ell_{\alpha} v_{\delta} \bar{v}_{\alpha}\right) \frac{C^{2}}{2^{11} \pi^{4} m_{0}^{4} G_{F}^{2}}\left\{2\left|B+T_{1}\right|^{2}+\left|T_{1}\right|^{2}\right. \\
& \left.-8 \operatorname{Re}\left(B T_{2}^{*}\right)-12 \operatorname{Re}\left(T_{1} T_{2}^{*}\right)+\left[-\frac{8}{3}+8 \ln \frac{m_{\delta}^{2}}{4 m_{\alpha}^{2}}\right]\left|T_{2}\right|^{2}\right\} .
\end{aligned}
$$

The decay rate for $\mu \rightarrow 3 e$ from similar Lorentz structures was also calculated long ago in Ref [33]. Their result coincides with ours only upon including the $K$ term as shown in eq (31). This term was easily missed since it is apparently of a higher order in $m_{\alpha}^{2}$.

Finally, the process (1) also implies an effective interaction that can induce the muoniumanti-muonium oscillation:

$$
\mathscr{L}_{\mathrm{eff}}=-G_{M \bar{M}} \bar{\mu} \gamma^{\alpha} P_{L} e \bar{\mu} \gamma_{\alpha} P_{L} e
$$

where the effective Fermi constant is,

$$
\frac{G_{M \bar{M}}}{\sqrt{2}}=\frac{C \xi^{2}}{m_{0}^{2}(4 \pi)^{2}} \sum_{x, y=1}^{2} z_{\mu x} z_{e x}^{*} z_{\mu y} z_{e y}^{*} H\left(r_{x}, r_{y}\right) .
$$

\section{Numerical analysis}

Before we embark on numerical discussion, we make some general remarks on the results obtained so far. This will help us identify potentially interesting regions in parameter space. The starting point is the induced neutrino masses that set a basic constraint on the free parameters in the octet model. Since the neutrinos are extremely much lighter than the octet particles, the effects due to Yukawa couplings may be relevant only when the $\lambda$ parameter is tiny. With fixed neutrino and octet masses, we have $|z| \propto(\lambda C)^{-1 / 2}$, where $C$ is the dimension of the real representation to which the new colored particles belong. Therefore, a smaller $\lambda$ tends to enhance the box diagrams more than the radiative ones, while a larger representation tends 
to suppress the former while leaving the latter intact. But in practice the box diagrams never dominate leptonic transitions for perturbative $z$ parameters when the stringent constraints from muon decays are taken into account. As a matter of fact, we find that those constraints are so strong that the branching ratios of all rare tau decays are generally much below the level to be accessible in the near future even when some $z$ parameters have a magnitude of order one. This arises from the fact that the lepton mixing is very close to the tri-bimaximal pattern whose entries are either order one or zero. This feature generally carries over to the $z$ parameters though the latter involve other parameters. For the tri-bimaximal mixing, we have for $\mathrm{NH}$

$$
z_{\alpha 1}^{\mathrm{tb}}=\frac{t}{1+t^{2}} \sqrt{\frac{\lambda_{-}}{\mu_{1}}}\left(\begin{array}{c}
+\frac{2}{\sqrt{3}} e^{i \alpha} \\
+\frac{2}{\sqrt{3}} e^{i \alpha}+\frac{\eta}{\sqrt{2}} r_{v} \\
-\frac{2}{\sqrt{3}} e^{i \alpha}+\frac{\eta}{\sqrt{2}} r_{v}
\end{array}\right), z_{\alpha 2}^{\mathrm{tb}}=\frac{t}{1+t^{2}} \sqrt{\frac{\lambda_{-}}{\mu_{2}}}\left(\begin{array}{c}
+\frac{\eta}{\sqrt{3}} e^{i \alpha} \\
+\frac{\eta}{\sqrt{3}} e^{i \alpha}-\frac{2}{\sqrt{2}} r_{v} \\
-\frac{\eta}{\sqrt{3}} e^{i \alpha}-\frac{2}{\sqrt{2}} r_{v}
\end{array}\right),
$$

and for $\mathrm{IH}$

$$
z_{\alpha 1}^{\mathrm{tb}}=\frac{t}{1+t^{2}} \sqrt{\frac{\lambda_{-}}{\mu_{1}}}\left(\begin{array}{c}
+\frac{\eta}{\sqrt{3}} r_{v} e^{i \alpha}+\frac{2 \sqrt{2}}{\sqrt{3}} \\
+\frac{\eta}{\sqrt{3}} r_{v} e^{i \alpha}-\frac{\sqrt{2}}{\sqrt{3}} \\
-\frac{\eta}{\sqrt{3}} r_{v} e^{i \alpha}+\frac{\sqrt{2}}{\sqrt{3}}
\end{array}\right), z_{\alpha 2}^{\mathrm{tb}}=\frac{t}{1+t^{2}} \sqrt{\frac{\lambda_{-}}{\mu_{2}}}\left(\begin{array}{c}
-\frac{2}{\sqrt{3}} r_{v} e^{i \alpha}+\frac{\sqrt{2} \eta}{\sqrt{3}} \\
-\frac{2}{\sqrt{3}} r_{v} e^{i \alpha}-\frac{\eta}{\sqrt{6}} \\
+\frac{2}{\sqrt{3}} r_{v} e^{i \alpha}+\frac{\eta}{\sqrt{6}}
\end{array}\right)
$$

where $r_{v}=\sqrt{\lambda_{+} / \lambda_{-}}>1$ by definition and $\eta=\left(1-t^{2}\right) / t$ is complex.

Since the constraints from the decays $\mu \rightarrow e \gamma$, $3 e$ make the majority of parameter space practically inaccessible, we turn to consider the possibility that the dominant contributions to those processes may be cancelled between the two octet fermions. This will then impose a relation among various parameters. Since the tri-bimaximal pattern serves as an excellent approximation to the mixing matrix and simplifies the analysis considerably, we shall determine the regions in parameter space where the cancellation occurs for the pattern. This will be employed later as a guide to scan parameters in interesting intervals where some processes might be accessible without breaking the stringent bounds on the muon decays. In addition, we find that the heavy scalar limit $m_{0} \gg m_{1,2}$ is particularly interesting. In the limit the radiative decays are independent of the scalar mass while the pure box induced decays are power enhanced. This apparently strange behavior is of course due to the fixed neutrino masses. But we should also keep in mind that we cannot take the limit literally for numerical analysis since $z$ being proportional to $m_{0}$ would exceed the perturbative regime.

In the heavy octet scalar limit, $r_{x} \ll 1$, all loop functions are independent of $r_{x}$ except that $G_{1}\left(r_{x}\right)$ has a residual $\ln r_{x}$ dependence. The suppression of muon decays then simplifies to the $(\mu e)$ cancellation condition:

$$
z_{\mu 1}^{\mathrm{tb} *} z_{e 1}^{\mathrm{tb}}+z_{\mu 2}^{\mathrm{tb} *} z_{e 2}^{\mathrm{tb}}=0
$$

which involves the parameters $\alpha, r_{v}, \eta$, and $R=m_{1} / m_{2}$. In case A all leading terms are cancelled, while in case B there remain $\ln r_{x}$ terms from $G_{1}$ which are significant only when $m_{1,2}$ are well separated. Our later numerical discussion will not involve this situation. Eq (37) 
can be solved exactly. Consider the NH first. The existence of solutions to eq (37) requires that

$$
\cos (2 \alpha) \leq f(R), f(x)=\frac{1}{2 x}\left[\frac{3 r_{v}^{2}}{8 x}\left(1-x^{2}\right)^{2}-\left(1+x^{2}\right)\right] .
$$

For $R \notin\left(R_{-}, R_{+}\right)$, the above is automatically fulfilled while for $R \in\left(R_{-}, R_{+}\right)$it has to be checked. Here we have denoted $R_{ \pm}=\left[\sqrt{1+2 /\left(3 r_{v}^{2}\right)} \pm \sqrt{2 /\left(3 r_{v}^{2}\right)}\right]^{2}$, which are roughly 0.51 and 1.97 using the best-fit values for neutrino masses [34], $\Delta m_{\mathrm{sol}}^{2}=7.6 \times 10^{-5} \mathrm{eV}^{2},\left|\Delta m_{\mathrm{atm}}^{2}\right|=$ $2.4 \times 10^{-3} \mathrm{eV}^{2}$. Denoting $\eta=|\eta| e^{i \beta}$, the phase $\beta$ is determined uniquely for given $(\alpha, R)$ by

$$
\tan \beta=\frac{1-R}{1+R} \tan \alpha, c_{1}=\sqrt{6} r_{v}(1-R) \frac{\cos \beta}{\cos \alpha}<0
$$

and the real positive solutions for $|\eta|$ are

$$
|\eta|_{ \pm}=\frac{1}{2 R}\left[-c_{1} \pm \sqrt{c_{1}^{2}-16 R}\right]
$$

To each $\eta$ correspond two values of $t, t_{ \pm}=\frac{1}{2}\left[-\eta \pm \sqrt{\eta^{2}+4}\right]$. There are thus generally four solutions to the $(\mu e)$ cancellation condition in eq (37), which will be named $t_{1,2}$ (from $\left.|\eta|_{+}\right)$ and $t_{3,4}$ (from $|\eta|_{-}$). Note that $t_{1} t_{2}=t_{3} t_{4}=-1$. The relation is not accidental but reflects a symmetry in our parametrization in eq (11): when $t$ is replaced by $-1 / t$ our $z$ only flips its sign.

The IH case is solved similarly. The existence of solutions to eq (37) requires that

$$
\cos (2 \alpha) \leq g(R), g(x)=\frac{1}{2 x}\left[\frac{r_{v}^{2}\left(1-x^{2}\right)^{2}}{8\left(x-r_{v}^{2}\right)\left(1-r_{v}^{2} x\right)}-\left(x^{2}+1\right)\right]
$$

Defining $R_{ \pm}=R_{0} \pm \sqrt{R_{0}^{2}-1}$ with $R_{0}=\left(4 r_{v}^{4}+r_{v}^{2}+4\right) /\left(9 r_{v}^{2}\right)$ and noting $r_{v}^{2}>R_{+}>R_{-}>r_{v}^{-2}$, the requirement is automatically fulfilled for $R \notin\left(R_{-}, R_{+}\right)$while it has to be checked for $R \in$ $\left(R_{-}, R_{+}\right)$. The latter interval is very narrow in IH since $R_{-} \approx 0.985$ and $R_{+} \approx 1.015$. There are generally two solutions to $\eta$ (except at $R=r_{v}^{2}$ ),

$$
\eta_{ \pm}=\frac{1}{2} e^{i \beta}\left[-c_{1} \pm \sqrt{c_{1}^{2}-4 c_{0}}\right]
$$

where

$$
\begin{aligned}
& \beta=\arctan \left(\frac{R-1}{R+1} \tan \alpha\right) \in(-\pi / 2, \pi / 2), \\
& c_{1}=\frac{\sqrt{2} r_{v}(1-R)}{\left(r_{v}^{2}-R\right)} \frac{\cos \beta}{\cos \alpha}, c_{0}=\frac{4\left(r_{v}^{2} R-1\right)}{\left(r_{v}^{2}-R\right)} .
\end{aligned}
$$

The solutions for $t$ are also denoted by $t_{1,2,3,4}$ as in NH case.

We show in Fig. 4 the curves for the cancellation condition in eq (37) for both $\mathrm{NH}$ and IH cases. In the upper panels, the real $t$ parameter varies as a function of $R$ at $\alpha=0$. All of four solutions are shown $\left(t_{1(2)}\right.$ : upper (lower) solid curve, $t_{3(4)}$ : upper (lower) dotted). Since 

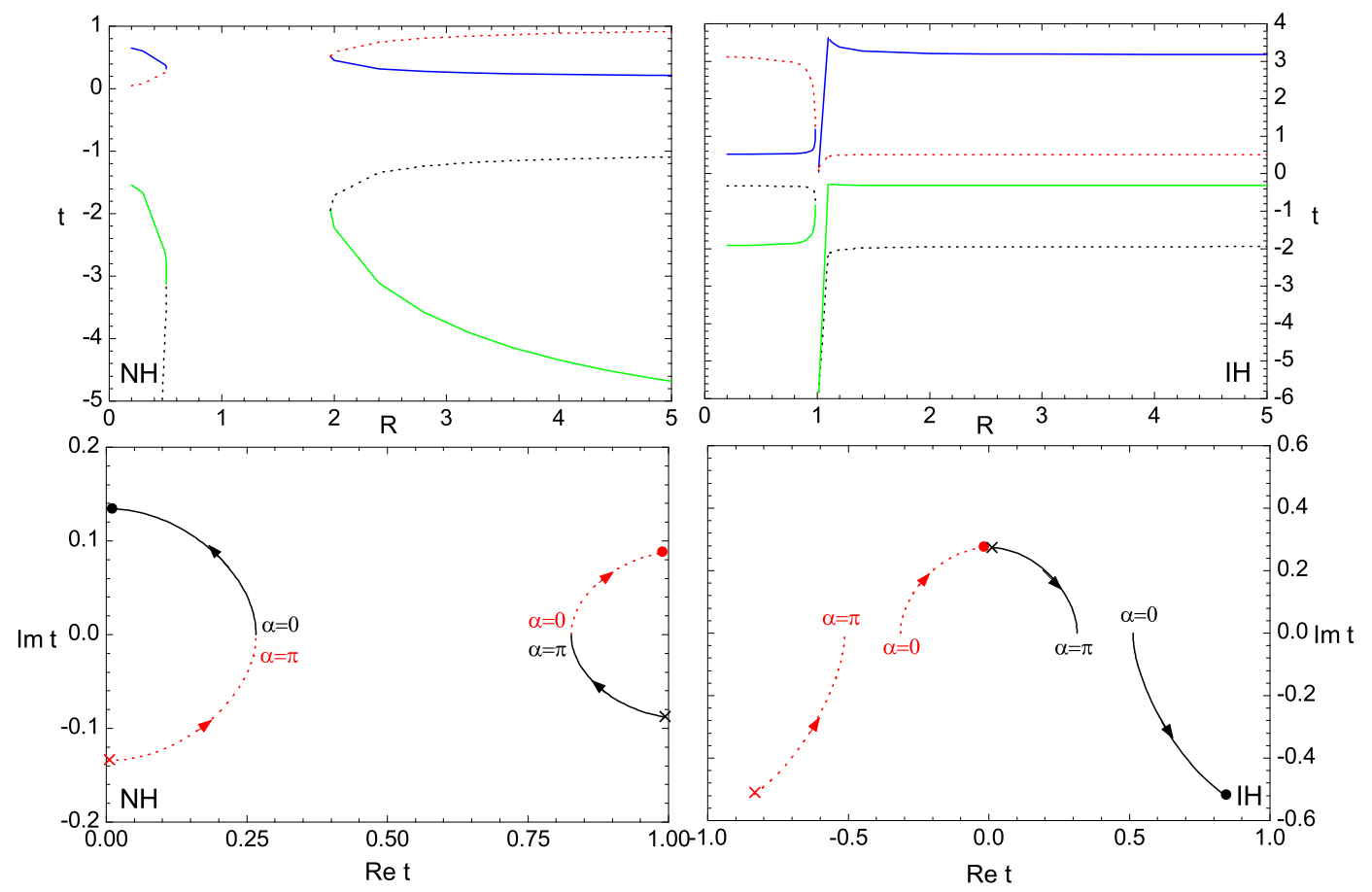

Figure 4: Curves for cancellation condition in eq (37) are shown for both NH and IH cases. Upper panels: real $t$ varies as a function of $R$ at $\alpha=0$. All four solutions are shown. Lower panels: real and imaginary parts of $t$ vary as $\alpha$ changes from 0 to $\pi$ at $R=3$. Only two out of four solutions are depicted. The starting and ending values of $\alpha$ are indicated. Note the discontinuity at $\alpha=\frac{1}{2} \pi$.

$|f(x)|<1$ for $x \in\left(R_{-}, R_{+}\right)$in the NH case, there are no solutions to $t$ when $R$ lies in the interval. The situation is similar in the IH case though the interval becomes very narrow due to $r_{V} \approx 1$ and $t$ varies rapidly close to the ends of the interval. When $R$ is removed away from $R_{ \pm} \sim 1$, it is a good approximation to assume $r_{v}=1$, in which limit the four solutions become flat in $R$ and saturate the values, $t=(-1 \pm \sqrt{3}) / \sqrt{2}, \sqrt{2} \pm \sqrt{3}$. In other words, at these values of $t$ the cancellation occurs independently of $R$ as long as $R$ is not too close to unity. The lower panels show how the complex $t$ varies as the Majorana phase $\alpha$ moves from 0 to $\pi$ at $R=3$. For clarity of illustration we only show $t_{1}$ (solid) and $t_{3}$ (dotted) for the $\mathrm{NH}$ case while $t_{2,4}$ can be recovered from $-1 / t_{1,3}$; similarly, for the IH case only $t_{1,4}$ (solid and dotted) are depicted while the major portions of $t_{2,3}$ lie outside of the displayed regions of $t$. The curves are discontinuous at $\alpha=\frac{1}{2} \pi$, jumping from one segment of a curve from below and at $\alpha=\frac{1}{2} \pi$ (indicated in the figure by a dot) to the other above the value (indicated by a cross).

Keeping in mind the cancellation curves we now display some results on the radiative and leptonic decays. For definiteness, the octet masses are always chosen to be

$$
m_{0}=10 \mathrm{TeV}, m_{1}=400 \mathrm{GeV}, m_{2}=200 \mathrm{GeV},
$$

while the $\lambda$ parameter is chosen such that the strict bounds on $\mu \rightarrow e \gamma, 3 e$ decays still leave some space in which tau decays are potentially accessible. We show in the upper panels of Fig. 
5 the branching ratios for $\mu \rightarrow e \gamma, 3 e$ as a function of $|t|$ at three points of $(\alpha, \arg t)=(0,0)$ (solid), $(\pi / 3,0.107)$ (dashed), and $(\pi / 3,0.485)$ (dotted) for the $\mathrm{NH}$ and tri-bimaximal mixing in case $\mathrm{A}$. We have assumed $\lambda=1.2 \times 10^{-9}$. Although the $t$ parameter is an independent complex parameter, we have chosen its argument properly for each value of $\alpha$ so that $|t|$ in the interval $[0,1]$ contains one or two points saturating the cancellation condition. At $R=2$ and $\alpha=0$, for instance, the cancellation occurs at $t_{1}=0.449, t_{3}=0.585$ plus the other two outside the interval, while at $\alpha=\pi / 3$ it occurs at $t_{1}=0.212 \exp (0.485 i), t_{3}=0.828 \exp (0.107 i)$ and two others. The current upper bounds on the decays [18, 19] are also indicated. It is clear that the allowed ranges of $|t|$ depend significantly on both $\alpha$ and $\arg t$. In the lower panels we show using the same parameters the branching ratios for $\tau \rightarrow e \mu, 3 \mu$ that are closest to the current upper bounds [20, 22]. Although their variations in $t, \alpha$ are not as strong as designed for the muon decays, they are still significant. At $(\alpha, \arg t)=(0,0)$ and $(\pi / 3,0.107)$ the branching ratio for $\tau \rightarrow 3 \mu$ can approach the bound in the allowed ranges of $|t|$. The results for other tau decays and anomalous magnetic moments are about two or more orders of magnitude smaller than their current bounds and are less sensitive to the parameters. Fig. 6 displays similar curves for IH at $\lambda=2.5 \times 10^{-9}$. The two curves in each panel correspond to $(\alpha, \arg t)=(0,0)$ (solid) and $(\pi / 3,-0.258)$ (dashed). The muon decays are significantly suppressed around $|t|=0.51$ and 0.61 respectively. Their sharper spikes can also be understood from the flatness of the $t-R$ cancellation curves in Fig. 3. In the allowed ranges of parameters the decay $\tau \rightarrow 3 \mu$ is a few times smaller than its upper bound in the most favorable situation, while other decays are largely unobservable.

The above results are presented for the simplified scenario of tri-bimaximal mixing. But global fittings of neutrino data generally prefer a slight deviation from it. We investigate now how rare decays could potentially be sensitive to the deviation. For definiteness, we fix $\theta_{12}$ and $\theta_{23}$ to the central values obtained in Ref. [34], $\sin ^{2} \theta_{12}=0.32, \sin ^{2} \theta_{23}=0.50$, and vary the small angle $\theta_{13}$ below its upper bound, $\sin ^{2} \theta_{13} \leq 0.05$. As we pointed out earlier, for arbitrarily chosen values of the new parameters $\alpha, t$ and masses $m_{0,1,2}$, all tau decays are generically too small to be observable when the bounds on rare muon decays are respected. Therefore, in the dominant portion of parameter space the decays cannot be sensitive to a small parameter like $\theta_{13}$. However, if the parameters happen to be located in the neighborhood of cancellation curves determined by eq (37) and exemplified in Fig. 4, the muon decays could be sensitive to $\theta_{13}$. We show in Fig. 7 how their branching ratios vary as a function of $\theta_{13}$ at $\lambda=10^{-8}$ and for case B. We have set $t=0.449$ (solid line) and 0.585 (dotted) for $\mathrm{NH}$ and $t=0.511$ for $\mathrm{IH}$. These values of $t$ correspond to the points at which significant cancellation takes place in case A for the tri-bimaximal mixing. It is interesting that $\operatorname{Br}(\mu \rightarrow 3 e)$ in $\mathrm{NH}$ reaches its minimum not at $\theta_{13}=0$ but around $\theta_{13} \sim 0.025$. For such a 'large' value of $\lambda$ other decays are simply not observable. 


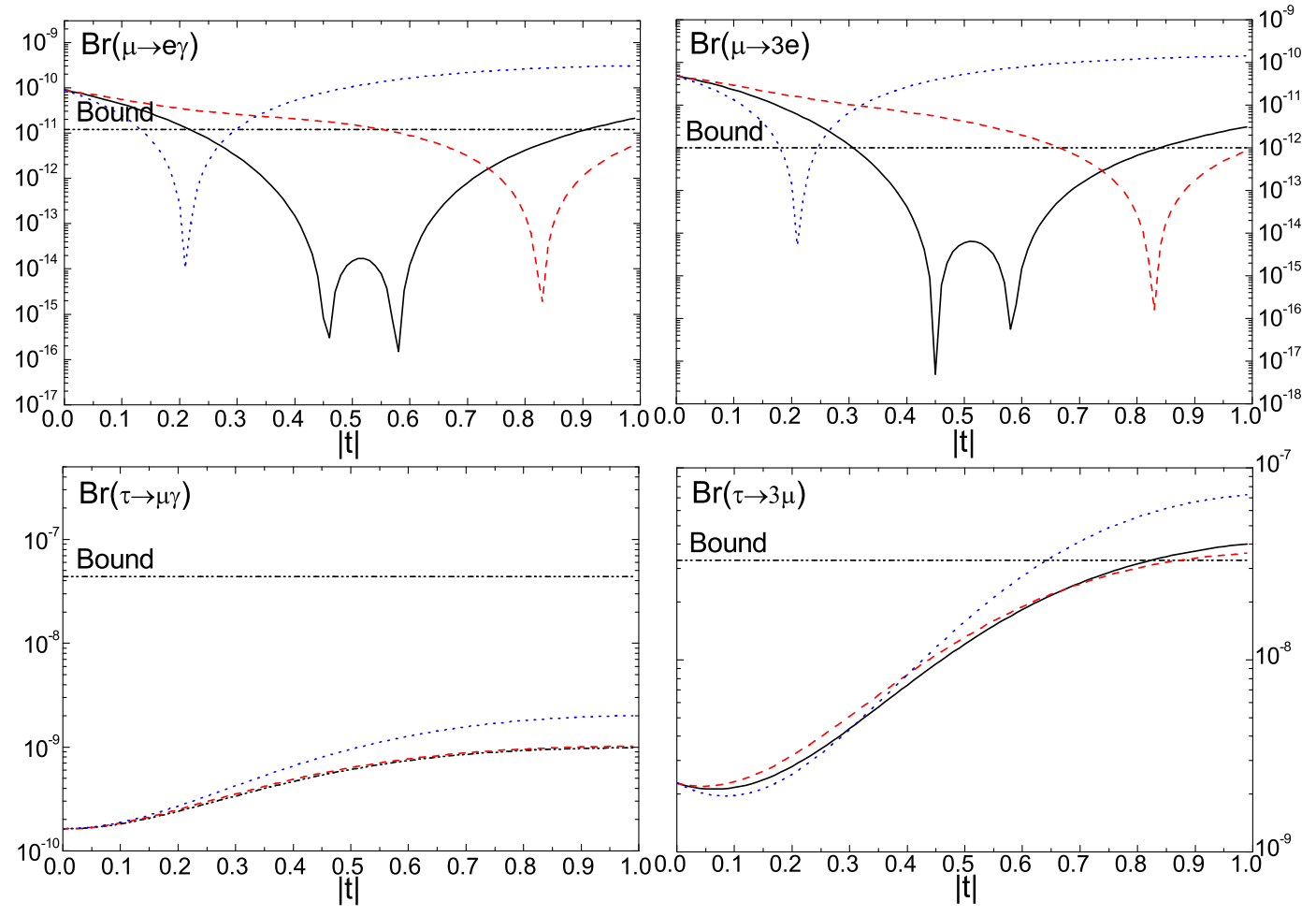

Figure 5: Branching ratios as a function of $|t|$ in $\mathrm{NH}$, tri-bimaximal mixing, and case A.

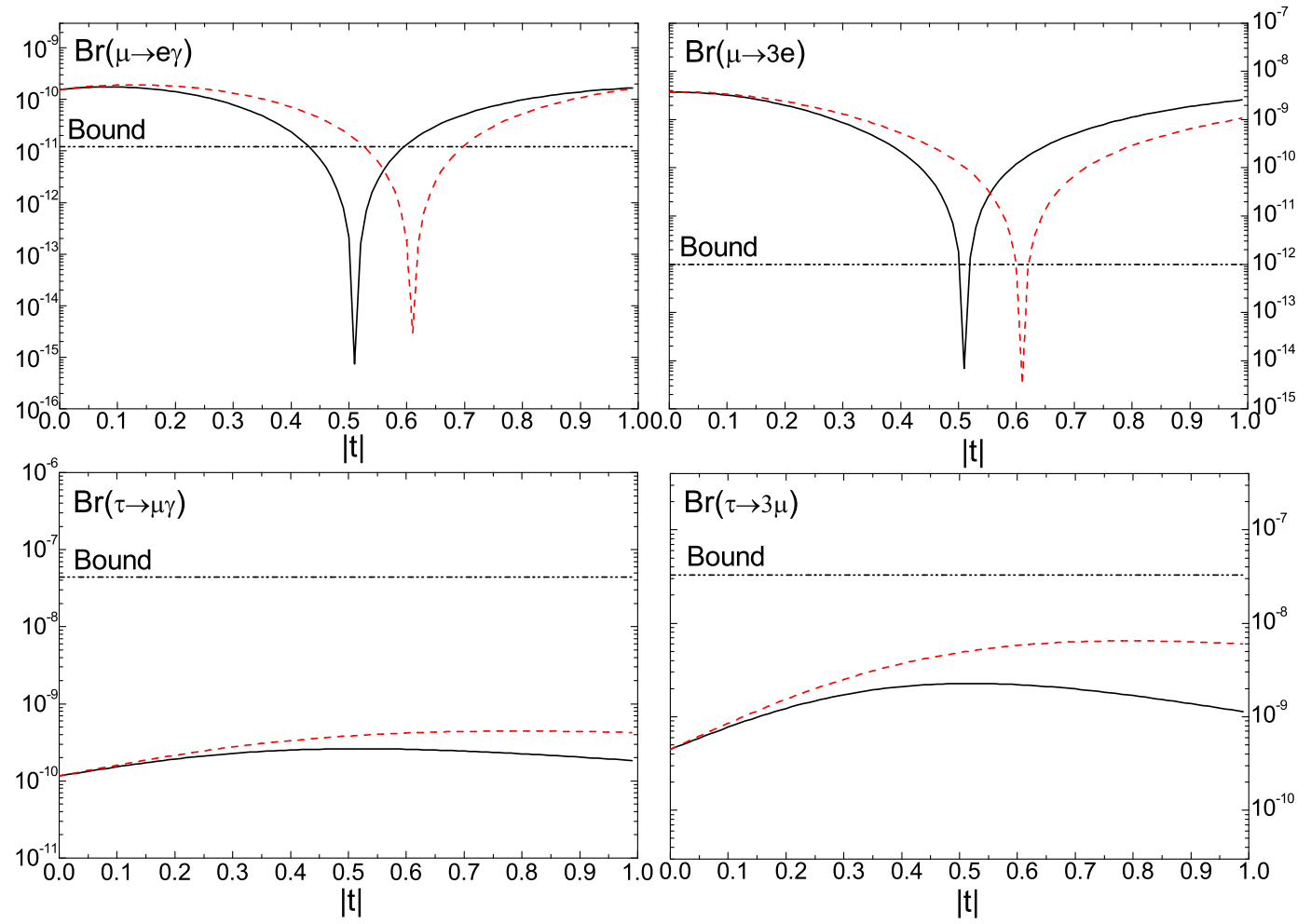

Figure 6: Branching ratios as a function of $|t|$ in $\mathrm{IH}$, tri-bimaximal mixing, and case A. 

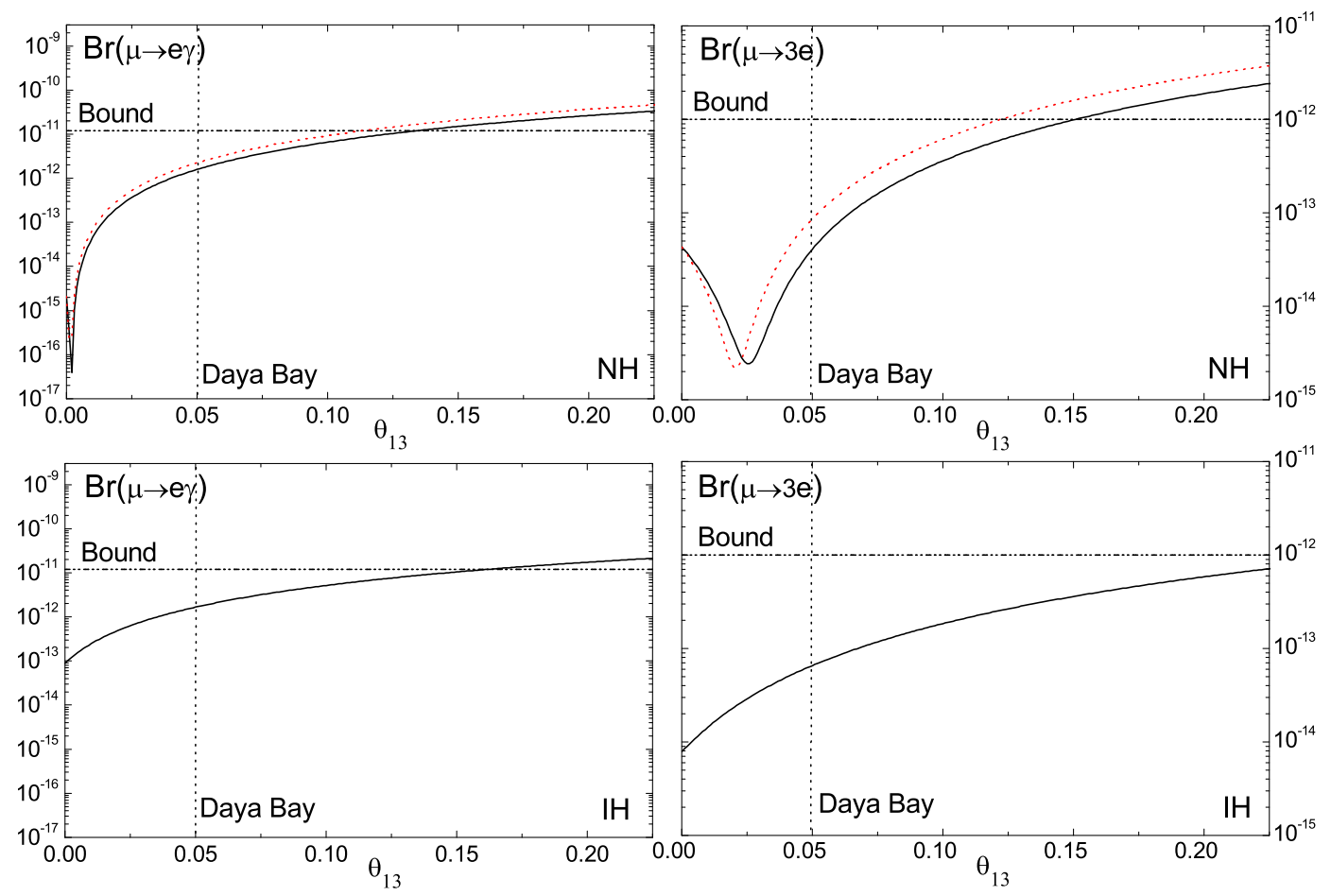

Figure 7: Branching ratios in case $\mathrm{B}$ as a function of $\theta_{13}$ at $\sin ^{2} \theta_{12}=0.32, \sin ^{2} \theta_{23}=0.50, \delta=$ 0 and $\alpha=0$. Upper panels for $\mathrm{NH}$ and lower ones for $\mathrm{IH}$. The proposed limit to be reachable in Daya Bay experiment is also indicated.

\section{Conclusion}

A mechanism that generates tiny neutrino mass and large lepton mixing generically induces lepton flavor violating transitions in the charged lepton sector, if there is any observable effect at all of the mechanism at low energies. The transitions are extremely suppressed by tiny neutrino masses if they are induced only by standard gauge interactions. Their observability would thus necessitate the existence of new particles that could interact with neutrinos and charged leptons separately. A model of this sort has been devised recently [11]. Its main merit is that the particles responsible for the interactions are colored, so that they could be copiously produced at hadron colliders. However, to make contact with the origin of neutrino mass it would be necessary to observe lepton signals produced from those colored particles. A realistic estimate of the signals should take into account the constraints that are already available. In this work we have made a complete analysis of the lepton flavor structure, provided a convenient parametrization to it, and studied systematically the radiative and pure leptonic transitions of the muon and tau leptons. We found that the current bounds on rare muon decays set a stringent constraint on rare tau decays that are generically far below the current experimental sensitivity for the majority of parameter space. However, there still exists the possibility that the new particles interfere destructively in the $(e \mu)$ sector such that the muon decays are significantly suppressed while their interactions with leptons are not necessarily reduced. This is indeed also 
the region in parameter space relevant to collider physics. We have explored this possibility for rare tau decays and showed that the decays $\tau \rightarrow \mu \gamma, 3 \mu$ are much enhanced compared to the general case. For the normal hierarchy of neutrino masses, the branching ratio for $\tau \rightarrow 3 \mu$ could reach the current level of precision while for the inverted hierarchy it is a few times smaller. Considering the rapid progress made in rare tau decays it is worthwhile pursuing further effects of this parameter region.

\section{Acknowledgement}

This work is supported in part by the grants NCET-06-0211, NSFC-10775074 and NSFC10975078.

\section{Appendix A: Phase space integrals}

We discuss and calculate some phase space integrals for the three-body decay $\ell_{\delta}(p) \rightarrow$ $\ell_{\alpha}\left(k_{1}\right) \ell_{\beta}\left(k_{2}\right) \bar{\ell}_{\gamma}\left(k_{3}\right)$. Its amplitude $\mathscr{A}$ originates from box diagrams and also from radiative transitions when a pair of $\ell$ and $\bar{\ell}$ is connected to the virtual photon, i.e., when $\alpha=\gamma$, or $\beta=\gamma$, or $\alpha=\beta=\gamma$. For our purpose of estimating LFV branching ratios, it is a good approximation to treat the final state leptons as massless. But one must be careful with the terms related to radiative transitions when doing algebra in $|\mathscr{A}|^{2}$. Although the logarithmic mass singularity, $\ln \left(m_{\delta}^{2} / m_{\gamma}^{2}\right)$, can be readily isolated, it is easy to miss some regular terms by setting masses (of leptons connected to the photon) to zero too early.

The spin-summed and -averaged decay rate is

$$
\Gamma=\frac{1}{1+\delta_{\alpha \beta}} \frac{1}{2 m_{\delta}} \operatorname{PS}_{3} \sum \overline{|\mathscr{A}|^{2}}
$$

where

$$
\mathrm{PS}_{3}=\int \frac{d^{3} \mathbf{k}_{1}}{(2 \pi)^{3} 2 E_{1}} \int \frac{d^{3} \mathbf{k}_{2}}{(2 \pi)^{3} 2 E_{2}} \int \frac{d^{3} \mathbf{k}_{3}}{(2 \pi)^{3} 2 E_{3}}(2 \pi)^{4} \delta^{4}\left(p-k_{1}-k_{2}-k_{3}\right)
$$

Using the kinematic symmetry in the final state, all required integrals can be converted to the following ones:

$$
\begin{aligned}
\left(p^{2}\right)^{(2,2,3)} I_{(1,2,3)} & =\operatorname{PS}_{3}\left(p \cdot k_{1}, k_{23}, k_{1} \cdot p k_{23}\right) \\
\left(p^{2}\right)^{(1,2)} J_{(1,2)} & =\mathrm{PS}_{3} \frac{\left(k_{1} \cdot p, k_{12} k_{31}\right)}{s_{23}} \\
p^{2} K & =\mathrm{PS}_{3} \frac{m_{2}^{2} p \cdot k_{1}}{s_{23}^{2}}
\end{aligned}
$$

where $k_{i j}=k_{i} \cdot k_{j}$ and $s_{23}=\left(k_{2}+k_{3}\right)^{2}$. The integrals $J_{1,2}, K$ appear only in radiative dipole transition terms, i.e., those proportional to $\left|T_{2}\right|^{2}$. Our convention here is that the virtual photon is connected to the leptons $\ell_{\beta}\left(k_{2}\right)$ and $\bar{\ell}_{\gamma}\left(k_{3}\right)$ with $\beta=\gamma$. 
The basic integrals are,

$$
\begin{aligned}
8 \pi Q^{2} \int \frac{d^{3} \mathbf{k}_{2}}{(2 \pi)^{3} 2 E_{2}} \int \frac{d^{3} \mathbf{k}_{3}}{(2 \pi)^{3} 2 E_{3}}(2 \pi)^{4} \delta^{4}\left(Q-k_{2}-k_{3}\right) & =\sqrt{\lambda\left(Q^{2}, k_{2}^{2}, k_{3}^{2}\right),} \\
96 \pi \int \frac{d^{3} \mathbf{k}_{2}}{(2 \pi)^{3} 2 E_{2}} \int \frac{d^{3} \mathbf{k}_{3}}{(2 \pi)^{3} 2 E_{3}}(2 \pi)^{4} \delta^{4}\left(Q-k_{2}-k_{3}\right) k_{2}^{\alpha} k_{3}^{\beta} & =g^{\alpha \beta} Q^{2}+2 Q^{\alpha} Q^{\beta},
\end{aligned}
$$

with $\lambda(a, b, c)=a^{2}+b^{2}+c^{2}-2 a b-2 b c-2 c a$. To integrate over $\mathbf{k}_{1}$, we determine the interval of its magnitude to be given by

$$
\left|\mathbf{k}_{1}\right|=\frac{1}{2} x m_{\delta}, x \in\left[0, x_{0}\right], x_{0}=1-4 \eta_{2}, \eta_{2}=\frac{m_{2}^{2}}{m_{\delta}^{2}} .
$$

Note that it is safe to set $m_{1}=0$ in computing the integrals $J_{1,2}, K$ even for the process (3). This is because the singularities in $s_{23}$ and $s_{31}$ do not overlap, as we pointed out in the main text. The final results are

$$
\begin{aligned}
& (4 \pi)^{3} I_{1}=\frac{1}{12}+O\left(\eta_{2}\right), \\
& (4 \pi)^{3} I_{2}=\frac{1}{24}+O\left(\eta_{2}\right), \\
& (4 \pi)^{3} I_{3}=\frac{1}{96}+O\left(\eta_{2}\right), \\
& (4 \pi)^{3} J_{1}=\frac{1}{4}\left[-\frac{3}{2}+\ln \frac{1}{4 \eta_{2}}\right]+O\left(\eta_{2}\right), \\
& (4 \pi)^{3} J_{2}=\frac{1}{48}\left[-\frac{11}{6}+\ln \frac{1}{4 \eta_{2}}\right]+O\left(\eta_{2}\right), \\
& (4 \pi)^{3} K=\frac{1}{16}+O\left(\eta_{2}\right) .
\end{aligned}
$$

\section{References}

[1] S. Weinberg, Phys. Rev. Lett. 43, 1566 (1979).

[2] E. Ma, Phys. Rev. Lett. 81, 1171 (1998) [arXiv:hep-ph/9805219].

[3] M. Gell-Mann, P. Ramond, R. Slansky, in: D. Freedman, P. van Nieuwenhuizen (Eds.), Supergravity, North-Holland, Amsterdam, 1979, p.315; T. Yanagida, in: O. Sawada, A. Sugamoto (Eds.), Proceedings of the Workshop on Unified Theory and Baryon Number in the Universe, KEK, Japan, 1979; R.N. Mohapatra, G. Senjanovic, Phys. Rev. Lett. 44 (1980) 912.

[4] W. Konetschny and W. Kummer, Phys. Lett. B 70, 433 (1977); T. P. Cheng and L. F. Li, Phys. Rev. D 22, 2860 (1980); J. Schechter and J. W. F. Valle, Phys. Rev. D 22, 2227 (1980). 
[5] R. Foot, H. Lew, X. G. He and G. C. Joshi, Z. Phys. C 44, 441 (1989).

[6] A. Zee, Phys. Lett. B 93, 389 (1980) [Erratum-ibid. B 95, 461 (1980)].

[7] A. Zee, Nucl. Phys. B 264, 99 (1986).

[8] K. S. Babu, Phys. Lett. B 203, 132 (1988).

[9] M. Aoki, S. Kanemura and O. Seto, Phys. Rev. Lett. 102, 051805 (2009) [arXiv:0807.0361 [hep-ph]].

[10] K. S. Babu, S. Nandi and Z. Tavartkiladze, arXiv:0905.2710 [hep-ph].

[11] P. Fileviez Perez and M. B. Wise, Phys. Rev. D 80, 053006 (2009) [arXiv:0906.2950 [hep-ph]].

[12] A. V. Manohar and M. B. Wise, Phys. Rev. D 74, 035009 (2006) [arXiv:hep-ph/0606172].

[13] For a recent phenomenological analysis to the octet scalars, see: C. P. Burgess, M. Trott and S. Zuberi, JHEP 0909, 082 (2009) [arXiv:0907.2696 [hep-ph]].

[14] R. S. Chivukula and H. Georgi, Phys. Lett. B 188, 99 (1987); G. D’Ambrosio, G. F. Giudice, G. Isidori and A. Strumia, Nucl. Phys. B 645, 155 (2002) [arXiv:hep-ph/0207036].

[15] M. Losada and S. Tulin, arXiv:0909.0648 [hep-ph].

[16] B. C. Odom, D. Hanneke, B. D’Urso and G. Gabrielse, Phys. Rev. Lett. 97, 030801 (2006) [Erratum-ibid. 99, 039902 (2007)].

[17] G. W. Bennett et al. [Muon G-2 Collaboration], Phys. Rev. D 73, 072003 (2006) [arXiv:hep-ex/0602035].

[18] M. L. Brooks et al. [MEGA Collaboration], Phys. Rev. Lett. 83, 1521 (1999) [arXiv:hep-ex/9905013].

[19] U. Bellgardt et al. [SINDRUM Collaboration], Nucl. Phys. B 299, 1 (1988).

[20] B. Aubert [The BABAR Collaboration], arXiv:0908.2381 [hep-ex].

[21] K. Hayasaka et al. [Belle Collaboration], Phys. Lett. B 666, 16 (2008) [arXiv:0705.0650 [hep-ex]].

[22] G. Marchiori and f. t. B. Collaboration, arXiv:0909.3870 [hep-ex].

[23] Y. Miyazaki et al. [Belle Collaboration], Phys. Lett. B 660, 154 (2008) [arXiv:0711.2189 [hep-ex]]. 
[24] J. Hisano, T. Moroi, K. Tobe and M. Yamaguchi, Phys. Rev. D 53, 2442 (1996) [arXiv:hep-ph/9510309]; J. Hisano, T. Moroi, K. Tobe, M. Yamaguchi and T. Yanagida, Phys. Lett. B 357, 579 (1995) [arXiv:hep-ph/9501407].

[25] M. Kakizaki, Y. Ogura and F. Shima, Phys. Lett. B 566, 210 (2003) [arXiv:hep-ph/0304254]; E. J. Chun, K. Y. Lee and S. C. Park, Phys. Lett. B 566, 142 (2003) [arXiv:hep-ph/0304069].

[26] A. Abada, C. Biggio, F. Bonnet, M. B. Gavela and T. Hambye, Phys. Rev. D 78, 033007 (2008) [arXiv:0803.0481 [hep-ph]].

[27] A. Abada, C. Biggio, F. Bonnet, M. B. Gavela and T. Hambye, JHEP 0712, 061 (2007) [arXiv:0707.4058 [hep-ph]].

[28] J. P. Bu, Y. Liao and J. Y. Liu, Phys. Lett. B 665, 39 (2008) [arXiv:0802.3241 [hep-ph]]; P. Q. Hung, Phys. Lett. B 659, 585 (2008) [arXiv:0711.0733 [hep-ph]].

[29] P. Q. Hung, Phys. Lett. B 649, 275 (2007) [arXiv:hep-ph/0612004].

[30] S. R. Choudhury, A. S. Cornell, A. Deandrea, N. Gaur and A. Goyal, Phys. Rev. D 75, 055011 (2007) [arXiv:hep-ph/0612327]; M. Blanke, A. J. Buras, B. Duling, A. Poschenrieder and C. Tarantino, JHEP 0705, 013 (2007) [arXiv:hep-ph/0702136].

[31] Y. Kuno and Y. Okada, Rev. Mod. Phys. 73, 151 (2001) arXiv:hep-ph/9909265]; A. Masiero, S. K. Vempati and O. Vives, New J. Phys. 6, 202 (2004) [arXiv:hep-ph/0407325]; M. Raidal et al., Eur. Phys. J. C 57, 13 (2008) arXiv:0801.1826 [hep-ph]].

[32] For a recent review on the muon anomalous magnetic moment, see: F. Jegerlehner and A. Nyffeler, Phys. Rept. 477, 1 (2009) [arXiv:0902.3360 [hep-ph]].

[33] T. P. Cheng and L. F. Li, Phys. Rev. D 16, 1425 (1977).

[34] M. Maltoni, T. Schwetz, M. A. Tortola and J. W. F. Valle, New J. Phys. 6, 122 (2004) [arXiv:hep-ph/0405172]. 